DOI: $10.20472 /$ IAC.2019.049.032

\author{
KEITA NISHIO \\ Aoyama Gakuin University, Japan \\ TAKASHI KABURAGI \\ International Chirstian University, Japan \\ SATOSHI KUMAGAI \\ Aoyama Gakuin University, Japan \\ TOSHIYUKI MATSUMOTO \\ Aoyama Gakuin University, Japan \\ YOSUKE KURIHARA \\ Aoyama Gakuin University, Japan
}

\title{
CONSTRUCTION OF ANXIETY PROPAGATION NETWORK AND ESTIMATION METHOD OF STAI SCORE FOR OF THE ANXIETY PROPAGATION NETWORK AND ESTIMATION METHOD OF THE STAI SCORE FOR STATE ANXIETY BY THE NIRS
}

\begin{abstract}
:
Recently, research has been conducted to estimate STAI (State-Trait Anxiety Inventory) score, an index of stress evaluation, by NIRS (Near-Infrared Spectroscopy). However, few studies related to STAI have considered the network structure of the brain, despite the importance of analyzing how information is propagated through brain networks. In this study, we define an anxiety propagation network structure from cerebral blood flow, and propose an STAI score for a state anxiety estimation method based on the network.

In the proposed method, we assume that anxiety is propagated via an anxiety propagation network, and that cerebral blood flow in the prefrontal area is affected by this propagation. We construct a dynamic Bayesian anxiety propagation network based on blood flow measured by multi-channel NIRS in the prefrontal area. In the constructed network, anxiety is propagated between nodes, and dependence relationships between nodes are represented by edges. The degree of dependence between nodes is expressed as the probability. To decrease the effect of the distance between nodes, probabilities are weighted according to Euclidian distance, and features are calculated by summing the weighted probabilities for each node. Finally, the STAI score for state anxiety is estimated by applying the features to a multiple regression analysis as the explanatory variables.

We conducted the validation experiment with 24 male and 24 female subjects. All subjects were scored by the STAI test. After scoring, the subjects set the wearable topography with ten channels to the prefrontal area. Each subject was asked to rest for 120 seconds to measure blood flow. The dynamic Bayesian network was created for all 48 data points, and its features were calculated.

To evaluate effect to the blood flow due to gender and hormone difference, we compared four cases of the gender gap combination of the training data and test data for the multiple regression analysis. In all cases, the accuracy of the proposed method was evaluated by the correlation coefficient.

In the training data for males, the correlation coefficients for male and female test data were 0.66
\end{abstract}


and 0.63 , respectively. When training data were for females, the correlation coefficients for male and female test data were 0.52 and -0.65 . We found that when the training data were for males, the correlation coefficient was larger, even if the test data were for females.

\section{Keywords:}

prefrontal blood flow, Bayesian network, STAI, NIRS.

JEL Classification: I19 